\title{
ÉDITORIAL
}

\section{Quel mode d'assainissement doit-on adopter en milieu rural ?}

\author{
*
}

DENDANT UNE GRANDE PARTIE de ma carrière d'hydrologue, j'ai surveillé la qualité de nombreuses eaux souterraines captées, aussi bien par la Ville de Paris que par d'autres collectivités, des industriels, des particuliers. F'ai bien souvent observé des contaminations imputables à un manque total du souci de l'hygiène publique. Très souvent mes observations m'amenaient à penser que seul le développement de l'assainissement collectif en milieu rural permettrait de mettre fin à cet état de choses. Pour moi, il n'y avait pas de doute, c'était la seule solution. Il me faut bien le confesser, c'était une erreur de jeunesse... qui a duré bien longtemps, car ce n'est guère qu'après avoir largement dépassé la cinquantaine que j'ai révisé ma position.

C'était à l'époque où, pour tout le monde, ou peu s'en faut, l'assainissement collectif était l'avenir. Il était en effet très séduisant de penser que les eaux usées seraient collectées, rassemblées, traitées et ne seraient rendues au milieu extérieur que lorsqu'elles seraient devenues à peu près inoffensives. Idée louable mais que la réalité se chargea d'infirmer.

\section{- L'ASSAINISSEMENT COLLEGTIF N'EST PAS UNE PANAGÉ}

Une récente enquête de la S.A.F.E.G.E. publiée dans le "Bulletin de liaison du Groupe Cortambert » a montré que lorsque l'assainissement est effectué en système séparatif, de très nombreux branchements d'eaux usées ou résiduaires sont raccordés au réseau d'eaux pluviales, donc rejetées ensuite sans aucun traitement. Que peut-on faire contre de telles pratiques, qu'elles soient dues ou non à des erreurs ? A peu près rien avant longtemps, certainement.

Mais, en supposant que toutes ces eaux soient dirigées sur les stations de traitement, est-on certain qu'il en ira beaucoup mieux? Ces stations chargées d'éliminer la majeure partie de la pollution ne remplis- 


\section{ÉDITORIAL}

sent pas totalement leur rôle dans la plus grande partie des cas, sauf pour des stations bien conçues, bien gérées et dont la capacité est bien adaptée au volume à traiter. Fe n'irai pas jusqu'à dire que de telles stations sont très rares, mais elles sont loin de constituer la majorité. En outre, même en de telles occurrences, où l'abattement de la pollution physique et chimique correspond aux normes autorisant le rejet dans le milieu extérieur, la diminution de la contamination microbiologique, bien que très nette, n'est pas suffisante pour que l'effluent ne soit pas nocif de ce point de vue. Heureusement, bien souvent le milieu récepteur jouit d'un bon pouvoir auto-épurateur. Est-il toujours suffisant? Non, et l'on a pensé à pratiquer une désinfection des eaux avant restitution. Avouons-le, dans l'immense majorité des cas, cette opération n'est que symbolique. De nombreux essais sont en cours qui devraient donner satisfaction pour les stations importantes. Les petites collectivités auront-elles les moyens de se doter de dispositifs onéreux, auront-elles le personnel compétent pour que les appareils fonctionnent bien? Je n'en suis pas certain.

Mais, direz-vous, même si ces eaux ne sont pas parfaitement traitées, il y a tout de même une amélioration par rapport à ce qui existait auparavant où chacun disséminait ses déchets et déjections de-ci, de-là. Voire. Les cours d'eau et le sol possèdent un grand pouvoir d'épuration, mais ce pouvoir n'est pas infini, loin de là et lorsqu'on concentre en un même point une quantité de pollution supérieure à celle que peuvent résorber les microorganismes du milieu extérieur, ceux-ci sont pratiquement empoisonnés et il n'y a plus d'auto-épuration.

Face à ce danger, que faire? Améliorer les stations, leur rendement, leur gestion? Les Agences Financières de Bassin y ont pensé et elles ont mis sur pied des Services d'Assistance Technique chargés de contrôler le fonctionnement des stations et surtout de donner tous conseils nécessaires aux exploitants. On peut espérer que, de ce point de vue, on observera peu à peu une amélioration. Heureusement, car pour les cités de quelque importance on ne peut plus concevoir d'autre assainissement que collectif.

\section{— L'ASSAINISSEMENT PARTICULIER OU AUTONOME.}

\section{Le traitement des eaux usées}

Pour les habitations isolées, les petites agglomérations, en un mot, pour le milieu rural, peut-être existe-t-il d'autres solutions, préférables. C'est ce qu'ont pensé certains qu'au début on pouvait considérer comme des non-conformistes de l'assainissement. Ils ont cherché à améliorer les moyens mis à la disposition de tout un chacun à condition qu'il puisse disposer d'une certaine surface de sol, c'est-à-dire l'immense majorité des habitants des campagnes. Ces études ont lieu dans la plupart des pays du monde où l'on se livre à la recherche.

Depuis longtemps déjà, il existait des moyens de se débarrasser, à l'échelle familiale, des matières excrémentielles, sans trop de dommage pour les milieux extérieurs. le plus répandu de ces dispositifs, vous le connaissez bien, c'est la "fosse septique ». Oh, elle était loin de donner toute satisfaction. Mais savait-on bien l'utiliser? En outre, peut-être était-elle perfectible. D'aucuns affirmaient : "introduisez-y l'eau parcimonieusement", alors que d'autres disaient : "il faut beaucoup, beaucoup d'eau dans une fosse septique ». On a donc recherché qui avait raison et on s'aperçut que c'étaient les derniers. Les bactéries apportées par les matières fécales sont tellement nombreuses que les effets de dilution et d'élimination sont négligeables.

Mais les fosses ne recevaient et ne reģoivent encore le plus souvent que les seules matières excrémentielles. Or les eaux ménagères, avec la préparation des aliments, les restes de cuisine, etc. sont loin d'être inoffensives. Très fermentescibles en raison de leur teneur élevée en matières organiques diverses, elles polluent de par cet apport, mais aussi elles constituent un excellent milieu de développement de très nombreux micro-organismes. Les admettre dans les fosses septiques? Vous n'y pensez pas : les antiseptiques qu'elles véhiculent (eau de javel, en particulier) entraveront le bon fonctionnement des fosses. Mais alors, comment se fait-il 


\section{ÉDITORIAL}

que rejetées dans la nature elles y fermentent si facilement ont pensé les non-conformistes. Ils étudièrent alors le comportement de fosses septiques qui, en plus des excréments, recevaient toutes les eaux ménagères (toilette, cuisine, lavage des légumes...) et découvrirent que le rendement des fosses était un peu moins bon, mais qu'il restait très acceptable. On en était arrivé au concept des "fosses septiques toutes eaux" (à l'exclusion des eaux pluviales, tout au moins pour le moment).

Toutefois, on devait considérer que l'effuent de ces fosses était encore putrescible : les transformations qu'il a subies ont eu lieu en anaérobiose, uniquement. Un traitement aérobie complémentaire aurait peut-être une action bénéfique sur cette qualité ? Ainsi naquit l'idée de l'élément épurateur aérobie. Ce dispositif, installé après la fosse, améliora grandement la qualité. Mais il n'est pas parfait. Le débit des eaux de cuisine et surtout des eaux de toilette (douches, bains) et celui des appareils de lavage est sujet à de grandes variations. De par sa conception, la fosse n'y est que peu sensible. Il n'en est pas de même de l'épurateur aérobie. Plusieurs modifications sont proposées et l'on voit poindre des améliorations. Mais, même en l'état actuel, l'ensemble fosse septique et élément aérobie donne un effluent acceptable.

Ajoutons que la fosse septique n'est pas le seul dispositif. A l'échelle familiale on trouve encore la micro-station, qui fonctionne sur le principe de l'aération prolongée. A l'échelle d'un immeuble important ou d'un petit groupe d'immeubles, existent la fosse IMHOFF ou la mini-station.

\section{La restitution dans le milieu extérieur}

Que va-t-on faire des effluents? Actuellement, ils gagnent bien souvent, hélas, le fossé voisin. Cette solution est la plus facile, mais elle est bien souvent génératrice de nuisances, aussi faut-il, toutes les fois que la chose est possible, disperser ces eaux dans le sol. Comme nous l'avons déjà dit, celui-ci jouit d'un très grand pouvoir auto-épurateur : physique et chimique par adsorption, rétention et échanges d'ions, mais aussi microbiologique par fixation des micro-organismes sur les particules du sol et aussi grâce à sa microflore et sa microfaune.

Si donc on peut faire infiltrer lentement l'eau, elle pourra parfaire son épuration dans les couches du sol proches de la surface et elle ne polluera ou ne contaminera pas les circulations et nappes aquifères souterraines. Toutefois, l'effluent à faire infiltrer possède un pouvoir colmatant non négligeable, c'est pourquoi les dispositifs de restitution (épis, pattes d'araignées...) ont des dimensions importantes. $\mathcal{N u l}$ doute que les progrès à venir, en particulier en ce qui concerne l'élément épurateur aérobie, permettront de réduire ces dimensions.

\section{Les études nécessitées par la restitution dans le sol}

Face à la pollution dispersée du milieu rural, nous avons maintenant des armes bien plus efficaces que l'assainissement collectif. Mais il reste toutefois un autre problème : tous les sols ne conviennent pas pour cette restitution : certains sont imperméables, d'autres au contraire trop perméables pour que le processus d'auto-épuration ait le temps de jouer.

Décidées à réhabiliter l'assainissement autonome, les Autorités Administratives et plus particulièrement le Ministère de l'Environnement, par le truchement des Agences Financières de Bassin, ont entrepris de mener une action sur deux fronts : d'une part, dans une région témoin, mener une enquête approfondie sur l'aptitude des sols à l'infiltration, d'autre part étudier les améliorations à apporter aux dispositifs actuels. Il semble d'ailleurs que l'enquête sur l'aptitude des sols doive déboucher sur une notion nouvelle, celle de l'aptitude des sites. En effet, le sol n'est pas seul à prendre en considération : il peut avoir un pouvoir d'infiltration très convenable, mais reposer sur une couche imperméable située à trop faible profondeur; sa pente peut être trop forte; la nappe aquifère peut être trop proche ; la couverture d'un sous-sol karstique trop mince... Dans tous ces cas des solutions de rechange sont déjà proposées. 


\section{- CON G L U S I O N}

Ainsi qu'on le voit, un gros effort est entrepris pour réhabiliter et développer l'assainissement autonome. La raison en est simple, il se trouve que, pour une fois, la Santé publique et l'Economie se rejoignent : ce qui coûte le plus cher dans un réseau d'assainissement collectif, ce n'est pas la station de traitement, mais le réseau de collecte. En habitat dispersé, l'assainissement collectif revient bien plus cher à la collectivité que l'assainissement autonome bien conçu, lorsqu'il est possible. Et la collectivité, c'est vous, c'est moi.

Battant ma coulpe, j'ai donc totalement abandonné ce que je souhaitais autrefois. Fe suis maintenant un ferme partisan de l'assainissement autonome chaque fois qu'il est possible en fonction de l'habitat et des caractéristiques du site. La meilleure preuve, c'est que je collabore à l'étude en cours.

Puissé-je avoir convaincu le plus grand nombre d'entre vous dans cet éditorial qui, pour une fois, n'est pas directement scientifique, mais très terre à terre, ce qui est normal puisqu'il s'agit souvent de sol.

\section{L.P. MAZOIT}

